\title{
Maximum fractional energy transmissible over a linear dispersive medium
}

\author{
E. Caglioti \\ Dipartimento di Fisica, Università di Roma La Sapienza, Rome, Italy \\ B. Crosignani and P. Di Porto \\ Dipartimento di Fisica, Università dell'Aquila, L'Aquila, Italy
}

Received May 8, 1989; accepted September 22, 1989

\begin{abstract}
We investigate the problem of transmitting the maximum fractional energy over a given length of a linear dispersive medium (e.g., a single-mode optical fiber) once the input pulse duration and the output detection time are assigned.
\end{abstract}

The solution of a class of optimization problems in optics is based on the introduction of prolate functions. ${ }^{1}$ In this frame, the emphasis is placed on topics concerned with spatial distribution of radiant energy in connection with diffraction at a finite aperture, which include the analytic determination of the mode patterns in laser resonator theory and problems of optimum image formation and processing. ${ }^{2,3}$ In this Letter we investigate the complementary time-domain problem of maximizing the energy transmitted through a linear dispersive medium, which turns out to possess its solution in terms of the above-mentioned prolate functions. More precisely, we consider a signal propagating in the $z$ direction of a dispersive medium and possessing a finite duration $\Delta$ at $z=0$. We assume that, after a given traveled length $L$, the signal is detected by a quadratic detector collecting the transmitted energy over a time interval $\gamma$. Our problem is to determine the input signal giving rise to the maximum value of the ratio $U_{d} / U_{i}$ between the detected and the input energies.

To this aim, let us recall that the electric field $\mathbf{E}(\mathbf{r}, t)$ inside the dispersive medium can be written as

$$
\mathbf{E}(x, y, z, t)=\mathbf{E}_{0}(x, y) \exp \left[-i \beta\left(\omega_{0}\right) z+i \omega_{0} t\right] \Phi(z, t),(1)
$$

where $E_{0}(x, y)$ is the transverse field configuration relative to the medium considered (uniform for a bulk medium or coinciding with the appropriate mode for the single-mode fiber) and $\beta\left(\omega_{0}\right)$ is the propagation constant at the carrier average angular frequency $\omega_{0}$. By truncating the Taylor expansion of $\beta(\omega)$ around $\omega_{0}$ to include the second-order term, the modal amplitude $\Phi(z, t)$ obeys the differential equations ${ }^{4}$

$$
\left(\frac{\partial}{\partial z}+\frac{1}{v} \frac{\partial}{\partial t}\right) \Phi(z, t)-\frac{i}{2 A} \frac{\partial^{2}}{\partial t^{2}} \Phi(z, t)=0,
$$

where

$$
v=\left(\frac{\mathrm{d} \beta}{\mathrm{d} \omega}\right)_{\omega=\omega_{0}}^{-1}, \quad A=\left(\frac{\mathrm{d}^{2} \beta}{\mathrm{d} \omega^{2}}\right)_{\omega=\omega_{0}}^{-1}
$$

are the group velocity and the second-order group dispersion, respectively. The general solution of Eq. (2) is

$$
\begin{array}{r}
\Phi(z, t)=\left(-\frac{i A}{2 \pi z}\right)^{1 / 2} \int_{-\infty}^{+\infty} \exp \left\{i \frac{A}{2 z}\left[t^{\prime}-(t-z / v)\right]^{2}\right\} \\
\times \Phi\left(0, t^{\prime}\right) \mathrm{d} t^{\prime},
\end{array}
$$

from which it immediately follows that

$$
\begin{aligned}
U_{d}= & \int_{\frac{L}{v}-\frac{\gamma}{2}}^{\frac{L}{v}+\frac{\gamma}{2}} \mathrm{~d} t|\Phi(L, t)|^{2}=\frac{|A| \gamma}{2 \pi L} \int_{-\frac{\Delta}{2}}^{+\frac{\Delta}{2}} \mathrm{~d} t^{\prime} \int_{-\frac{\Delta}{2}}^{+\frac{\Delta}{2}} \mathrm{~d} t^{\prime \prime} \\
& \times \frac{\sin \left[\frac{A\left(t^{\prime}-t^{\prime \prime}\right) \gamma}{2 L}\right]}{\frac{A\left(t^{\prime}-t^{\prime \prime}\right) \gamma}{2 L}} \exp \left(i \frac{A t^{\prime 2}}{2 L}\right) \\
& \times \exp \left(-i \frac{A t^{\prime \prime 2}}{2 L}\right) \Phi\left(0, t^{\prime}\right) \Phi^{*}\left(0, t^{\prime \prime}\right) .
\end{aligned}
$$

Our purpose is to find the maximum value of the ratio $U_{d} / U_{i}$ as a function of the input signal, which is equivalent, by employing standard Lagrange multiplier technique, to solving the variational problem

$$
\begin{aligned}
\delta\left(U_{d}-\lambda U_{i}\right)= & \delta\left[\int_{\frac{L}{v}-\frac{\gamma}{2}}^{\frac{L}{v}+\frac{\gamma}{2}}|\Phi(L, t)|^{2} \mathrm{~d} t-\lambda\right. \\
& \left.\times \int_{-\frac{\Delta}{2}}^{+\frac{\Delta}{2}}|\Phi(0, t)|^{2} \mathrm{~d} t\right]=\delta\left[\int_{-\frac{\Delta}{2}}^{+\frac{\Delta}{2}} \mathrm{~d} t^{\prime}\right. \\
& \times \int_{-\frac{\Delta}{2}}^{+\frac{\Delta}{2}} \mathrm{~d} t^{\prime \prime} K\left(t^{\prime}-t^{\prime \prime}\right) \tilde{\Psi}\left(t^{\prime}\right) \tilde{\Psi}^{*}\left(t^{\prime \prime}\right) \\
& \left.-\lambda \int_{-\frac{\Delta}{2}}^{+\frac{\Delta}{2}} \mathrm{~d} t|\tilde{\Psi}(t)|^{2}\right]=0,
\end{aligned}
$$


where

$$
\begin{gathered}
K\left(t^{\prime}-t^{\prime \prime}\right)=\frac{\sin \left[\frac{|A|\left(t^{\prime}-t^{\prime \prime}\right) \gamma}{2 L}\right]}{\pi\left(t^{\prime}-t^{\prime \prime}\right)}, \\
\tilde{\Psi}(t)=\Phi(0, t) \exp \left(i \frac{A t^{2}}{2 L}\right),
\end{gathered}
$$

and $\lambda$ is the appropriate multiplier.

After introduction of the dimensionless time $\tau=t /$ $T$, where $T=2 L /|A| \gamma$, Eq. (6) yields the eigenvalue integral equation ${ }^{1}$

$$
\int_{-c}^{+c} \mathrm{~d} \tau^{\prime} \frac{\sin \left(\tau-\tau^{\prime}\right)}{\pi\left(\tau-\tau^{\prime}\right)} \Psi\left(\tau^{\prime}\right)=\lambda \Psi(\tau)
$$

where $\Psi(\tau)=\Psi(t / T)=\tilde{\Psi}(t)$ and

$$
c=\frac{\Delta}{2 T}=\frac{|A| \gamma \Delta}{4 L} \text {. }
$$

According to Eqs. (5), (8), and (9), $\lambda=\lambda(c)$ represents the ratio $U_{d} / U_{i}$, so that one has to look for the eigenfunction corresponding to the maximum value of $\lambda$. This problem has been extensively studied in Ref. 1 , and the solution turns out to be given by the linear prolate function of zero order $\Psi_{0}(\tau)$ [with associated eigenvalue $\lambda_{0}(c)$ ]. Therefore the optimum input signal is given by

$$
\hat{\Phi}(0, t)=\Phi_{0} \exp \left(-i A t^{2} / 2 L\right) \Psi_{0}\left(\frac{t}{T}\right),
$$

with the behaviors of $\Psi_{0}(\tau)$ and $\lambda_{0}(c)$ shown in Figs. 1 and 2 , respectively.

The above considerations can be readily extended to the case in which the carrier exhibits random fluctuations. To this end, one has to take into account the average usually performed by the detector over a time scale large compared with the coherence time of the carrier but still much smaller than $\gamma$. In our procedure this is done by replacing $|\Phi(z, t)|^{2}$ with $\left\langle|\Phi(z, t)|^{2}\right\rangle$, where the angular brackets stand for the short time average. After factorizing $\Phi(0, t)$ into a fast random part $F(t)$ and a slow deterministic part $S(t)$ associated with the modulation process, i.e.,

$$
\Phi(0, t)=F(t) S(t),
$$

the averaging operation leads to an integral equation analogous to Eq. (9) of the form

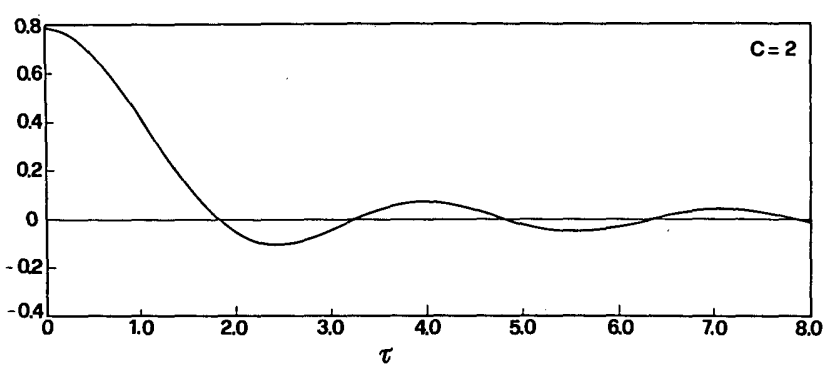

Fig. 1. Behavior of zero-order linear prolate function $\Psi_{0}(\tau)$ for $c=2$. Note that $-\Delta / 2<t<\Delta / 2$ corresponds to $-c<\tau<$ c.

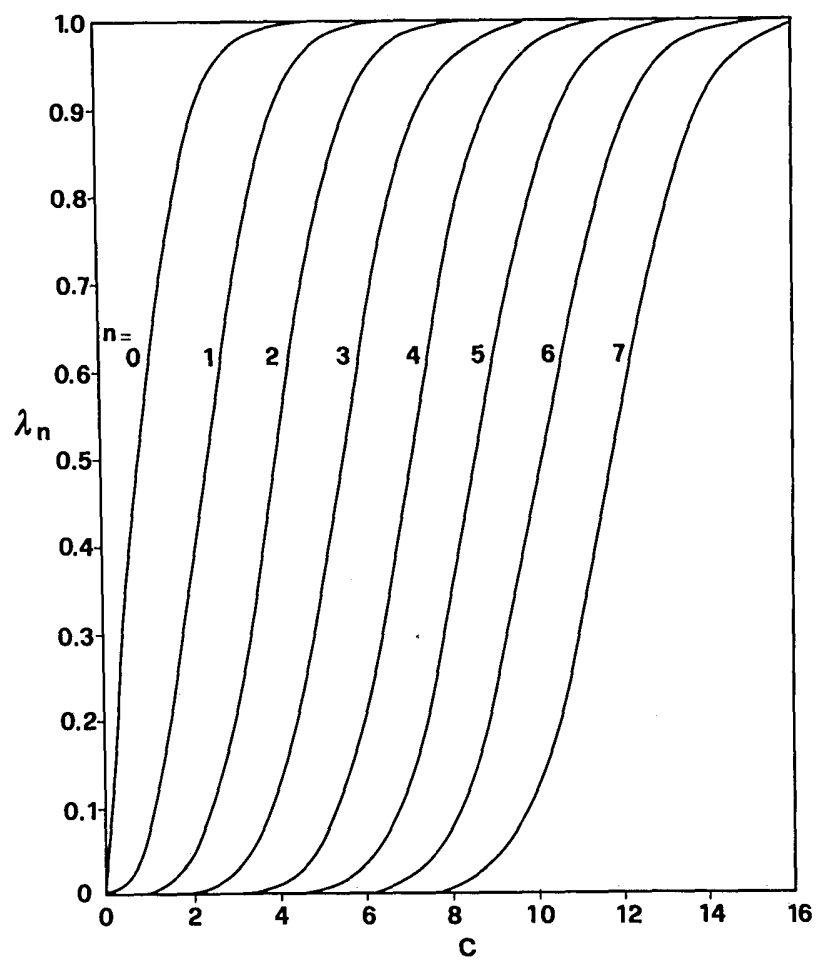

Fig. 2. Behavior of the lowest-order eigenvalues $\lambda_{n}$ as functions of $c$.

$$
\frac{1}{\pi} \int_{-\frac{\Delta}{2}}^{+\frac{\Delta}{2}} \mathrm{~d} t^{\prime} K\left(t-t^{\prime}\right) G\left(t-t^{\prime}\right) \tilde{S}\left(t^{\prime}\right)=\lambda \tilde{S}(t),
$$

where $G\left(t-t^{\prime}\right)$ is the normalized correlation function of the stochastic factor $F(t)$, i.e.,

$$
G\left(t-t^{\prime}\right)=\frac{\left\langle F(t) F^{*}\left(t^{\prime}\right)\right\rangle}{\left\langle|F(t)|^{2}\right\rangle} .
$$

Obviously, whenever the coherence time $T_{c}$ of the source is much larger than T, Eq. (13) reduces to Eq. (9). In general, Eq. (13) does not admit of known analytic solutions even for simple expressions of $G(t-$ $\left.t^{\prime}\right)$. In the limit $T_{c} \ll T$, it reduces to

$$
\frac{T_{c}}{\sqrt{\pi} T} \tilde{S}(t)=\lambda \tilde{S}(t),
$$

so that any function $\tilde{S}(t)$ is a solution with an eigenvalue $\lambda=T_{c} / \sqrt{\pi} T$.

In order to illustrate the order of magnitude of the quantities involved in our problem we note that the fundamental dimensionless parameter $c$ can be written as [see Eq. (10)]

$$
c=\frac{\gamma}{4 T_{\mathrm{cr}}},
$$

where $T_{\text {cr }}=L \delta \omega /|A|$ is the chromatic delay associated with the frequency bandwidth $\delta \omega=2 \pi / \Delta$. On the other hand, it is apparent from Fig. 2 that our optimization procedure has an effective meaning only for numerical values of $c$ not much larger than 1 . In effect, for $c \gg 1$, a large number of linear prolate functions possess an eigenvalue $\lambda_{n} \approx 1$, and their com- 
pleteness ensures energy optimization for any reasonable input signal [provided that it exhibits the appropriate frequency chirping required by Eq. (11)].

For a dispersive medium, we consider a single-mode fiber operating at $1.6 \mu \mathrm{m}$, where the chromatic delay is of the order of $20 \mathrm{psec} / \mathrm{km} \cdot \mathrm{nm}$ (see, e.g., Ref. 4), and $1200 \mathrm{~km}$ long. Under these conditions,

$$
T_{\mathrm{cr}}=\frac{1.6 \times 10^{-8}}{\Delta} \mathrm{psec}
$$

where $\Delta$ is expressed in seconds. If we assume that $\Delta$ $=0.1$ nsec, i.e., $T_{\mathrm{cr}}=160$ psec, Eq. (16) implies, for example, that a value $c=2$ can be obtained by choosing $\gamma \approx 1.3$ nsec. For the instantaneous linear frequency chirp required by Eq. (11), one has to sweep, over the initial time interval $\Delta$, a frequency range of

$$
\Omega=\frac{|A|}{L \Delta}=\frac{2 \pi}{T_{\mathrm{cr}}} \text {. }
$$

With the numerical values given above, this corresponds to $\Omega \approx 40 \mathrm{GHz}$, which is within the reach of the available laser-diode technology.

B. Crosignani and P. Di Porto are also associated with the Fondazione Ugo Bordoni, Rome, Italy. This research has been partially supported by the Italian Ministry of Education.

\section{References}

1. B. R. Frieden, in Progress in Optics, E. Wolf, ed. (NorthHolland, Amsterdam, 1971), Vol. 9, p. 311.

2. F. Gori and C. Palma, J. Phys A 8, 1709 (1975); P. De Santis and C. Palma, Opt. Acta 23, 743 (1976).

3. M. Bertero and E. R. Pike, Opt. Acta 29, 727 (1982).

4. S. Solimeno, B. Crosignani, and P. Di Porto, Guiding, Diffraction, and Confinement of Optical Radiation (Academic, New York, 1986), Chap. 8. 